\title{
Bounds on the range of density-functional-theory \\ point-defect levels in semiconductors and insulators
}

\author{
N. A. Modine, A. F. Wright, and S. R. Lee \\ Sandia National Laboratories, Albuquerque, New Mexico 87185-1315, USA \\ Corresponding Author Email: namodin@sandia.gov
}

\begin{abstract}
Defects in semiconductors and insulators are characterized by their levels, which are defined as the values of the Fermi level at which the charge state of the defect changes. Kohn-Sham density functional theory calculations for charged defects have been widely and successfully used to predict defect levels. Due to their lower computational cost and demonstrated ability to predict levels spanning the measured band gap, semilocal exchange-correlation functionals are widely used in these calculations. However, there is a potential pitfall in using semilocal functionals: Although they often predict accurate energies for adding or removing electrons from states that are localized near the defect, the famous band gap error results in overly small energies for adding or removing electrons from extended band edge states. As a result, electrons (or holes) that should occupy localized states may become partially or fully delocalized. In order to help detect and analyze such cases, we introduce bounds on the defect levels that can be obtained using a given functional, supercell, and Brillouin zone sampling. Since these bounds correspond to the charge transition levels of the corresponding defect-free supercell, comparison with the bounds reveals when a calculated level is behaving in a bulk-like rather than defect-like manner. We find that the bounds depend significantly on supercell
\end{abstract}


size due to band-filling effects that arise from the finite charge density created when one electron is added to or removed from a finite-sized supercell, and this size dependence helps explain the success of defect level calculations using semilocal functionals.

\section{Introduction}

Point defects in semiconductors and insulators may exist in more than one charge state. The possible charge states of a defect and its levels, which are defined as the values of the Fermi level at which the defect charge state changes, are technologically important because they control the relative rates of carrier capture and emission, and thereby determine how much influence the defect has on carrier recombination and minoritycarrier device performance. Measurements of defect levels are challenging, and using experiments to identify the atomistic origin (e.g., vacancy, interstitial, antisite, etc.) of a level can require a lifetime of work. Consequently, there has been considerable interest in theoretical calculations of defect levels.

The most widely used approach to calculating defect levels relies on Kohn-Sham density functional theory (DFT). ${ }^{1}$ Typically, calculations are performed in a parallelpiped (hereinafter referred to as a supercell) containing a defect surrounded by bulk material. Integer numbers of electrons are added to or subtracted from the supercell to produce a set of candidate charge states, and results from DFT calculations for these candidates are used to identify the valid charge states and compute the associated defect levels. The main approximation in Kohn-Sham DFT is the functional used to quantify electron exchange and correlation energies. At first glance, frequently used semilocal exchangecorrelation functionals such as the Local Density Approximation (LDA) and Generalized 
Gradient Approximation (GGA) appear to be poorly suited for defect studies because they yield band gaps - the difference in the conduction-band-minimum eigenvalue (conduction-band edge, CBE) and the valence-band-maximum eigenvalue (valence-band edge, VBE) - that are often less than half of the measured values. Since the Fermi level, which determines a defect's charge state and defines its levels, lies within the band gap in undoped material, it follows that the range of defect levels will be limited by the band gap. Thus, one might expect the LDA and GGA band gap problem to seriously degrade the accuracy of DFT defect levels.

Recent calculations comparing semilocal functionals with the more computationally expensive, and generally more accurate, hybrid functionals have shown that the semilocal and hybrid functionals typically give similar results for the spacing between defect levels. 2,3 These results help explain the success of defect level calculations using semilocal functionals. In contrast, the valence band edge (VBE) and conduction band edge (CBE) eigenvalues shift dramatically when different functionals are used. The difference between the VBE and CBE eigenvalues is the Kohn-Sham band gap. Typically with semilocal functionals, this difference is too small (the famous band gap problem), with the VBE positioned too high, and the CBE positioned too low, relative to defect levels aligned with respect to the average electrostatic potential. ${ }^{2,3}$ These errors in the band edges lead to a situation in which a level that lies within the band gap with hybrid functionals may lie within one of the band edges in semilocal calculations. When this happens, calculations using semilocal functionals typically have occupied conduction band states or de-occupied valence band states for one of the charge states involved in that level. 
When a defect level calculation involves delocalized band edge states, as described above, the results may fail to represent the intended transition between localized charge states of the defect. ${ }^{4}$ Therefore, it is important to identify when the results of a calculation are characteristic of delocalized rather than localized charges. To date, methods aimed at identifying this situation have not been methodically studied. The most typical approach for addressing this question has been to look for a delocalized component in charge density differences ${ }^{4}$ or frontier wavefunctions (often projected into atomic-like orbitals to allow convenient inspection). Alternatively, the Kohn-Sham energy levels of the defect-containing cell can be compared to the levels obtained in bulk material. More recently, plots of the Kohn-Sham levels as a function of inverse cell size have been used to identify when a defect related Kohn-Sham eigenvalue approaches the bulk VBE or CBE as the cell size increases. ${ }^{4}$

Although the above approaches can be useful, they do not directly address the usual quantities of interest in DFT calculations for defects: the defect levels and formation energies. With current techniques, it is usually difficult to reach a definitive conclusion as to whether the defect levels and formation energies from a given calculation can be trusted. It is tempting to reject categorically all calculated levels that lie below the bulk VBE or above the bulk CBE, but this would severely limit the applicability of semilocal functionals to defect calculations and contradict a long history of success in using such functionals. If two charge states have different structures, the associated defect level may exceed the CBE by as much as the Frank-Condon relaxation energy before any loss of charge to the conduction band. The analogous statement holds for levels below the VBE. Even when delocalization does occur, we will show that the occupation of band edge 
states can be sufficiently small that the calculated level is not substantially compromised. In fact, the movement of a defect level through a bulk band edge eigenvalue is only accompanied by an abrupt transition from a fully localized to a fully delocalized charge distribution in the limit of infinite supercells.

The purpose of this paper is to seek a systematic and unambiguous method for identifying when calculated defect levels become inaccurate due to the involvement of delocalized band edge states. For a given exchange-correlation functional, supercell, and Brillouin zone sampling, the range of defect levels is limited not by the Kohn-Sham band gap, but instead the defect levels are bound between the energies to add an electron to the finite volume of bulk material surrounding the defect and remove an electron from it. To a good approximation, these energies can be evaluated for a defect-free bulk supercell having the same size as the supercell in which the defect calculations are performed, and thus the same bounds can be used for all defects in a given material. Since the bounds represent the energetic cost of adding or removing an electron in the bulk supercell, a calculated defect level must increasingly involve delocalized band edge states as it approaches a bound. In particular, when a defect level turns and follows a bound as a function of some parameter such as supercell size, we know that the calculated defect level no longer represents the intended transition between localized charge states of the defect.

For the case of an infinite bulk system, Mori-Sanchez, Cohen, and Yang ${ }^{5}$ discuss the electron-addition and electron-removal energies (our bounds) and refer to their difference as the fundamental gap. Here we extend their work to finite-sized bulk supercells, and we will refer to the resulting difference, which gives the range of DFT defect levels in a 
finite supercell, as the supercell-size-dependent (SSD) gap. Latham et al. have discussed these same size-dependent quantities in the context of aligning defect levels to experimental band edges. ${ }^{6}$ For semilocal functionals such as the LDA and GGA, the SSD gap is equal to the Kohn-Sham band gap in the limit of an infinite-sized supercell. However, we find in this work that the SSD gap increases monotonically with decreasing supercell size and becomes comparable to the measured band gap for supercell sizes typically used in DFT defect calculations. Thus, the maximum possible range of DFT defect levels will depend on the size of the supercell used in the DFT calculations (and the Brillouin zone sampling) but will be larger than the Kohn-Sham band gap. While this result may seem surprising, we show that it is due to the same band-filling effects responsible for the apparent increase in the band gaps measured in optical absorption experiments in degenerately doped semiconductors.

We develop our bounds analysis of calculated defect levels as follows: In Section 2, we set the stage for our work by reviewing the standard approach to DFT defect calculations and explaining the relationship between the present work and this standard approach. Then, in Section 3, we derive bounds on defect levels in infinite- and finitesized supercells and present a practical procedure for calculating these bounds. We end Section 3 by discussing the relationship between the supercell-size-dependence of the bounds and band-filling effects. We proceed in Section 4 by giving three examples for specific defects where we compare DFT-based defect levels to the bounds. Such comparisons are shown to reveal if a calculated defect level involves transitions between well-localized defect charge states or instead involves carriers in band-edge-like states. In 
Section 5, we conclude with emphasis on how the bounds can be practically applied to improve DFT calculations of defect levels.

\section{Standard approach for DFT defect levels}

To begin, we review the approach used in typical DFT calculations of defect levels, wherein one finds the minimum total energy $E^{D}(q, L)$ of defect $D$ in charge state $q$ using a supercell of size $L$ containing the defect surrounded by bulk material. To obtain the defect levels, a range of different charge states and possibly a range of different supercell sizes are considered. An energy minimization is performed with respect to the ionic coordinates of the system, where the minimization may involve both a relaxation of coordinates for a particular defect structure and a comparison of different structures. Typically, periodic boundary conditions are applied to the supercell, effectively producing an infinite periodic array of defects, and a Brillouin zone sampling scheme is chosen. In order for the Poisson equation to have a well-defined solution, the total charge within the supercell must be neutral. To obtain a neutral supercell when treating a defect in non-zero charge state $q$, many researchers add a spatially uniform, compensating charge density with an integrated charge $-q .{ }^{7}$ Others modify the charge distribution and electrostatic potential in more complex ways to remove some of the long-ranged interactions between a charged defect and its periodically repeated replicas. ${ }^{8,9,10,11,12}$ The defect results reported herein were obtained using a uniform compensating background charge density.

For a given defect structure and supercell size, the formation energy of the defect is given by the well-known expression ${ }^{13,14,15}$ 


$$
E_{f}^{D}\left(q, L, E_{F}\right)=E^{D}(q, L)-E^{B}(0, L)-\sum_{i} n_{i} \mu_{i}+q E_{F},
$$

where $E^{D}(q, L)$ is the defect total energy defined above, $E^{B}(0, L)$ is the total energy of the neutral host supercell, $n_{i}$ denotes the number of atoms of type $i$ that are added to $\left(n_{i}>0\right)$ or removed from $\left(n_{i}<0\right)$ the bulk supercell to form the defect, and $\mu_{i}$ is the chemical potential of each reservoir providing atomic exchange. The Fermi level, $E_{F}$, is defined in terms of the reference chemical potential of the reservoir providing electron exchange, $\mu_{\mathrm{e}}$, and a relative Fermi level, $\varepsilon_{F}$, such that $E_{F} \equiv \mu_{\mathrm{e}}+\varepsilon_{F}$. Given Eq. (1), the thermodynamic level for a transition between defect charge states $q-1$ and $q$ is defined as the Fermi level where the formation energies for these states are equal: ${ }^{13}$

$$
\Delta^{D}(q-1 / q, L)=\left[E_{f}^{D}\left(q-1, L, E_{F}=\mu_{e}\right)-E_{f}^{D}\left(q, L, E_{F}=\mu_{e}\right)\right] .
$$

Equation (2) is often rewritten in terms total supercell energies as ${ }^{3,6}$

$$
\Delta^{D}(q-1 / q, L)=E^{D}(q-1, L)-E^{D}(q, L)-\mu_{e} .
$$

In the remainder of this paper, we will adopt the convention $\mu_{\mathrm{e}}=0$ (and not the traditional convention $\mu_{\mathrm{e}}=\varepsilon_{\mathrm{VBE}}$, where $\varepsilon_{\mathrm{VBE}}$ is the energy of the bulk valence band maximum). ${ }^{6}$ This choice simply shifts all band edges and levels by a constant value to suppress the usual trivial constant reference energy $\left(\varepsilon_{\mathrm{VBE}}\right)$ that would otherwise appear in many of the equations to be presented below. Importantly, no attempt to realign or post-process bands or levels is being made at this point.

As discussed in the recent review article by Freysoldt et al. ${ }^{13}$ and in a recent paper by Komsa et al., ${ }^{4}$ separate a posterior procedures are typically used to correct the defecttransition levels and defect-formation energies given above by Eqs. (1-3) for finite-size supercells. The purpose of these corrections is to remove the effect of artificial interactions between defects that arise from the large defect concentrations inherent in 
methods utilizing periodic finite-size supercells. ${ }^{4,13}$ By applying these corrections, one obtains calculated defect properties in the dilute limit that are suitable for comparison to experiments.

While corrections for a number of different physical effects may be important for typical finite-size supercells (for instance, wavefunction-overlap effects, elastic interactions, and magnetic interactions), ${ }^{13}$ the most important corrections for charged defects are typically electrostatic. These extensively researched electrostatic corrections are typically separated into two distinct, but related, contributions arising from (1) spurious electrostatic interaction of the charged defect with its periodically repeated images and with the constant-density neutralizing background charge, $4,8,9,10,11,12,13,16,17,18,19,20,21$ and (2) the lack of an absolute reference for the long-range electrostatic potential, ${ }^{22}$ which drives the need for procedures to align the average electrostatic potential of the defect-containing supercell with the potential of the defectfree bulk supercell. ${ }^{4,13,21,23,24,25}$ Additional procedures intended to accurately align the defect levels even when the exchange-correlation functional leads to significant errors in the band edges have been developed. ${ }^{2,3,26}$ In cases where comparisons have been made between theory and experiment, defect levels calculated by DFT (using periodic finitesize supercells and then corrected a posterior in the manner just described) often agree with experimental levels to within a few tenths of an electron volt $(\mathrm{eV}){ }^{2,26,27,28,29,30} \mathrm{Of}$ particular interest herein, such calculations have been shown in several cases to predict defect levels with an energy range comparable to the measured band gap of the material even when the Kohn-Sham band gap is much smaller than the measured band gap. 
The present work is not an attempt to further refine these important electrostatic correction and alignment methods, which already rest in many capable hands throughout the field. The bounds that we derive apply to the unprocessed levels given in Eq. 3 and are independent of subsequent analysis. The role of these bounds is to help determine when further processing of the defect energies and/or levels obtained from a given calculation is justified. Depending on whether or not calculated levels behave independently of the bounds, we will separate the levels into localized defect levels, which are suitable for additional processing, and band-edge-related levels, which fail to satisfy the assumptions required for further processing. Since the hydrogenic wavefunctions associated with shallow defect levels are composed of a superposition of band edge states, they will follow the bounds and be assigned to the latter category. Although such levels become loosely localized around the defect in a sufficiently large cell, they differ from our localized defect levels in important aspects such as scaling with supercell size and sensitivity to the exchange correlation functional.

Our categorization of levels can be extended to separate charge states into localized defect charge states and band-edge-related charge states. If the calculated $\Delta^{D}(q-$ $1 / q, L)$ level follows the upper bound, the $q-1$ charge state is a band-edge-related charge state with an electron in the conduction band, while if the calculated $\Delta^{D}(q-$ $1 / q, L)$ level follows the lower bound, the $q$ charge state is band-edge-related charge state with a hole in the valence band. If both $\Delta^{D}(q-1 / q, L)$ and $\Delta^{D}(q / q+1, L)$ are localized defect levels, we can conclude that the q charge state is a localized defect charge state without significant occupation of the conduction band or de-occupation of the valence band. 
It is important to note that our categorization of levels and charge states is a property of a particular calculation using a specific exchange correlation functional, supercell size, and Brillouin zone sampling, and thus it has no direct relationship to the question of whether a given defect level or charge state is physically valid. Answering this latter question requires applying the correction procedures discussed above to determine the infinite supercell-size limit of the defect level and align it with the experimental band edges. Then, the level is predicted to be valid if the fully corrected result lies within the experimental band gap. The importance of distinguishing localized defect levels from band-edge-related levels rests with the fact that the existing correction schemes, so critical to calculation of the dilute-limit properties of a defect, generally assume that the excess charge in a charged defect calculation is localized near the defect. Thus, these corrections must be applied only to localized defect levels and charge states, while setting aside calculations that give band-edge-related results. Otherwise, misleading or incorrect results may be obtained in the sought-after dilute limit.

\subsection{Supercell-size-dependent bulk levels}

In analogy with the discussion of defect levels in Section 2, we can define chargestate transition levels for defect-free bulk material when one electron is added to or removed from a bulk supercell along with a uniform compensating background charge density. Consider a bulk supercell of size $L$, where $E^{B}(0, L)$ is the total energy of the neutral supercell, $E^{B}(-1, L)$ is the total energy of this supercell with one electron added, and $E^{B}(+1, L)$ is the total energy with one electron removed. The energy to add one electron is given by 


$$
\Delta^{B}(-1, L)=E^{B}(-1, L)-E^{B}(0, L),
$$

and the energy to remove one electron is given by

$$
\Delta^{B}(+1, L)=E^{B}(0, L)-E^{B}(+1, L) .
$$

Drawing upon the definition of the fundamental gap given in Ref. ${ }^{5}$, we can now define the supercell-size-dependent (SSD) gap introduced above,

$$
\mathrm{E}_{G A P}^{B}(L)=\Delta^{B}(-1, L)-\Delta^{B}(+1, L)=E^{B}(-1, L)+E^{B}(+1, L)-2 E^{B}(0, L) .(6)
$$

For a finite system, a free molecule for example, with zero boundary conditions on the electrostatic potential at infinite distance, $\Delta^{B}(-1, L)$ would be the negative of the calculated electron affinity and $\Delta^{B}(+1, L)$ would be the negative of the calculated ionization energy. ${ }^{5}$ In the case of a periodically repeated supercell, $\Delta^{B}(-1, L)$ and $\Delta^{B}(+1, L)$ have an analogous meaning, but an arbitrary integration constant appears in the definition of the electrostatic potential. Using typical normalization conventions for plane-wave DFT calculations, $\Delta^{B}(-1, L)$ and $\Delta^{B}(+1, L)$ are often positive values. This reflects the fact that the vacuum level in a plane-wave DFT calculation does not correspond to zero potential, and the vacuum level should be subtracted from $\Delta^{B}(-1, L)$ and $\Delta^{B}(+1, L)$ in order to recover fully the interpretation of these quantities as the negative of the electron affinity and ionization energy.

We will now consider the infinite supercell-size limits of $\Delta^{B}(-1, L)$ and $\Delta^{B}(+1, L)$. The DFT total energy is a linear function of charge between integer charge values for the exact DFT functional, and as shown in Ref. ${ }^{5}$, this is true even for approximate exchangecorrelation functionals such as the LDA and GGA in the infinite system size limit. In addition, such semilocal functionals lack a derivative discontinuity, ${ }^{31,32}$ and as a result, the slope of this linear function is given by the Kohn-Sham eigenvalue of the partially 
filled orbital. Consequently, we obtain the infinite supercell-size limits $\Delta^{B}(-1, \infty)=$ $\varepsilon_{C B M}^{B}$ and $\Delta^{B}(+1, \infty)=\varepsilon_{V B M}^{B}$, where $\varepsilon_{C B M}^{B}$ and $\varepsilon_{V B M}^{B}$ are the Kohn-Sham CBE and VBE, respectively. ${ }^{5}$ In the infinite supercell-size limit, Eq. (6) becomes

$$
\mathrm{E}_{G A P}^{B}(\infty)=\Delta^{B}(-1, \infty)-\Delta^{B}(+1, \infty)=\varepsilon_{C B M}^{B}-\varepsilon_{V B M}^{B},
$$

and the SSD gap is equal to the Kohn-Sham band gap. Therefore, like the Kohn-Sham band gap, the SSD gap is severely underestimated in the infinite supercell-size limit when using typical (LDA and GGA) exchange-correlation functionals. In contrast, it will be shown in Section 3.3 that the SSD gap in finite-sized supercells is larger than the KohnSham band gap thereby allowing the range of DFT defect levels to exceed the KohnSham band gap.

\subsection{Bounds on defect levels in the infinite supercell-size limit}

To set up our consideration of bounds on defect levels in finite-sized supercells, we turn first to a formal derivation of bounds on the defect levels when the levels are computed with DFT in the infinite supercell-size limit using a given exchange-correlation functional. Using the framework established by Eqs. (1)-(7), we consider a system consisting of a pair of similar but independent supercells, one containing a defect surrounded by bulk material with charge state $q$ and the other containing neutral bulk material. Now, we add one electron to either the defect supercell or the bulk supercell. In the infinite supercell-size limit, the interaction between a defect and a delocalized band edge state vanishes (because the average distance between the defect and the electron is infinite). If the interaction between the defect and the added electron is repulsive, the extra electron will occupy such a delocalized state when added to either supercell, and the 
combined energy of the system will be independent of which supercell contains the added electron. On the other hand, if the interaction between the defect and the added electron is attractive, it is energetically favorable for the electron to localize near the defect, and the combined energy of the system will be lower when the electron is located in the defect supercell. In terms of total energies, these considerations give the expression

$$
E^{D}(q-1, \infty)+E^{B}(0, \infty) \leq E^{D}(q, \infty)+E^{B}(-1, \infty),
$$

which is easily rearranged to get the bound

$$
\Delta^{D}(q-1 / q, \infty) \equiv E^{D}(q-1, \infty)-E^{D}(q, \infty) \leq E^{B}(-1, \infty)-E^{B}(0, \infty) \equiv \Delta^{B}(-1, \infty) .
$$

A similar argument in which we instead remove an electron from (or add a hole to) either the defect supercell with charge $q-1$ or the bulk supercell gives

$$
E^{D}(q, \infty)+E^{B}(0, \infty) \leq E^{D}(q-1, \infty)+E^{B}(+1, \infty),
$$

which again is easily rearranged to get the bound

$$
\Delta^{D}(q-1 / q, \infty) \equiv E^{D}(q-1, \infty)-E^{D}(q, \infty) \geq E^{B}(0, \infty)-E^{B}(+1, \infty) \equiv \Delta^{B}(+1, \infty) .
$$

Taken together, Eqs. (9) and (11) show that in the infinite supercell-size limit, the calculated defect levels should be bound between the energies of removing an electron from and adding an electron to the bulk material. Given the infinite supercell-size limits discussed above for semilocal functionals, we arrive at the following formal bounds for the infinite supercell-size limit:

$$
\varepsilon_{V B M}^{B} \leq \Delta^{D}(q-1 / q, \infty) \leq \varepsilon_{C B M}^{B} .
$$

These bounds apply to any given defect and charge-state transition. We may conclude that the range of calculated defect levels obtained using semilocal functionals 
should indeed be rigorously constrained to the Kohn-Sham band gap in the infinite supercell-size limit. Since the correct range of actual defect levels should be limited to the much larger measured band gap, this conclusion at first suggests that there would be serious errors in many of the levels calculated by DFT. However, DFT defect levels are not obtained from calculations in infinite-sized supercells. Instead, calculations are performed for supercells containing approximately 100 to 1000 atoms, where the computational effort is currently manageable. The as-calculated results are then postprocessed to remove interactions between defects in different supercells in order to estimate the defect level (or the defect formation energy) in the infinite supercell-size limit.

\subsection{Bounds on defect levels in finite-sized supercells}

To understand how this use of finite-sized supercells influences calculated defect levels, we propose that the rigorous bounds given in Eq. 9 and Eq. 11 for infinite-sized supercells transform to approximate bounds for finite-sized supercells:

$$
\Delta^{B}(+1, L)-\delta(L) \leq \Delta^{D}(q-1 / q, L) \leq \Delta^{B}(-1, L)+\delta(L)
$$

where $\delta(L)$ is some relatively small energy that vanishes in the infinite supercell-size limit. The approximate nature of these bounds arises because an electron or hole in a delocalized band-edge state of a finite-sized supercell does not have enough freedom to completely avoid a repulsive interaction with the defect. However, it can largely avoid the high-energy region near the defect leading to only a small interaction, which is given by $\delta(L)$. 
To understand the implications of the approximate bound proposed in Eq. (13), we used DFT to calculate $\Delta^{B}(+1, L)$ and $\Delta^{B}(-1, L)$ for a range of bulk GaAs supercell sizes. The calculations were performed with the Socorro code, ${ }^{33}$ using a plane-wave basis with a 40 Rydberg cutoff to represent the Kohn-Sham orbitals, norm-conserving pseudopotentials (NCPs), and the LDA ${ }^{1,34}$ for exchange and correlation. The NCPs were constructed using the fhi98PP code ${ }^{35}$ with three electrons treated as valence $\left(4 \mathrm{~s}^{2}\right.$ and $\left.4 \mathrm{p}^{1}\right)$ for $\mathrm{Ga}$ and five electrons treated as valence $\left(4 \mathrm{~s}^{2}\right.$ and $\left.4 \mathrm{p}^{3}\right)$ for As.

In addition to the supercell size, the bounds $\Delta^{B}(+1, L)$ and $\Delta^{B}(-1, L)$ depend on the Brillouin zone sampling used in the calculations. For example, if only the $\Gamma$ point is used to sample the Brillouin zone, the bounds are almost independent of supercell size and remain very close to the VBE and CBE. This behavior is demonstrated in Fig. 5 of Ref. 36. On the other hand, if a limited number of k-points are used, and the k-point grid is displaced off the $\Gamma$ point, as in Refs. 2, 4, and 28, or the occupations of the Kohn-Sham orbitals are modified so that each k-point has the same total occupation, as in Ref. 27, it is possible to further open the gap between the bounds for small to moderate sized supercells. However, in the limit of large supercells, all k-points fold into a vanishingly small Brillouin zone volume, and the bounds will still converge toward the VBE and CBE eigenvalues. Although we believe that our bounds analysis could provide interesting insights into the effectiveness of these alternative Brillouin zone sampling schemes, we have adopted the traditional approach to Brillouin zone sampling in our calculations. Well-converged Monkhorst-Pack ${ }^{37}$ meshes were used to sample the Brillouin zone, and occupations of the Kohn-Sham orbitals were calculated using a Fermi distribution with $\mathrm{kT}=1.877 \times 10^{-3}$ Ryd (room temperature). 
Test calculations for the equilibrium zinc-blende structure predicted a $10.595 \mathrm{Bohr}$ lattice constant and a 0.720 Mbar bulk modulus. Consistent with the general trends observed when using the LDA, the lattice constant is $0.8 \%$ smaller and the bulk modulus is $4.8 \%$ larger than measured values.

Different supercells of a periodically repeated crystal correspond to the same physical system and give the same energy per volume when the calculations are converged with respect to Brillouin zone sampling. Thus, the energetic effects of adding an electron to a large supercell can be evaluated by adding the same density of electrons to a small supercell. The same principle holds for removing an electron from a large supercell. Thus, the bounds for large supercells can be obtained efficiently from calculations for a small supercell. To obtain bounds in cubic target supercells containing $N=8,64,216,512,1000,1728,2744,4096,5832,8000,27,000,64,000$ atoms, we performed calculations in a neutral 8-atom supercell and in 8-atom supercells with excess electronic charge $q= \pm 8 / N$. For each supercell, the density of the Monkhorst-Pack sampling mesh was increased until the bounds were converged to within $1.3 \mathrm{meV}$.

Figure 1 shows plots of the bounds vs. the electron density, $|q| / V$, added to (removed from) the target supercell. A striking feature in these plots is the density-dependent difference between the SSD gap (the upper bound minus the lower bound) and the KohnSham band gap. As expected from Section 3.1, the SSD gap is closest to the Kohn-Sham band gap $(\approx 0.1 \mathrm{eV}$ larger $)$ for the largest target supercells, and it appears to be approaching the Kohn-Sham band gap as the density decreases. For consistency with our defect calculations, the occupations of the Kohn-Sham orbitals were computed using Fermi smearing at room temperature, and this leads to an additional small upward 
(downward) offset of $\leq 0.04 \mathrm{eV}$ in the upper (lower) bound. For the smallest two target supercells, the SSD gap is close to or exceeds the measured band gap, $1.42 \mathrm{eV} .{ }^{38}$ In the next-larger target supercell (containing 216 atoms) - which is widely used in present-day calculations of defect levels - the SSD gap, and thus the possible energy range of calculated defect levels, is $85 \%$ of the measured band gap and $70 \%$ larger than the KohnSham band gap. We will discuss the implications of this increased energy range in Section 4.

To understand the difference between the SSD gap and the Kohn-Sham band gap, we first note that the deviation of the upper bound from the Kohn-Sham CBE is significantly larger than the deviation of the lower bound from the Kohn-Sham VBE. This difference in behavior between the two bounds suggests band-filling effects because such effects are inversely proportional to the effective carrier mass, and the effective electron mass is much smaller than the effective hole mass in GaAs. With this in mind, we calculated Burstein-Moss shifts ${ }^{39}$ for GaAs as a function of carrier density using band-edge effective electron and hole masses obtained from parabolic fits of the DFT bandstructure at the $\Gamma$-point in the Brillouin zone. The calculated masses were suitably averaged to account for anisotropic band shapes and light/heavy hole degeneracy. ${ }^{40}$ Our computed effective masses along [001], [011], and [111] directions are similar to previous spinless DFT results in GaAs. ${ }^{41}$ Reduction of the results to their single-band equivalents for use in the Burnstein-Moss equation yields effective electron and hole masses of $\mathrm{m}_{\mathrm{e}}{ }^{*} / \mathrm{m}_{0}=0.039$ and $\mathrm{m}_{\mathrm{h}}{ }^{*} / \mathrm{m}_{0}=1.10$, respectively.

Comparison of the resulting Burstein-Moss shifts with the bounds (Fig. 1) provides quantitative and convincing evidence that band filling is responsible for the charge 
density-dependence of the bounds. At relatively ordinary densities $\left(<10^{19} \mathrm{~cm}^{-3}\right)$ where the Burstein-Moss shifts are most applicable, they are in good agreement with the bounds. At a density of approximately $2 \times 10^{19} \mathrm{~cm}^{-3}$, where the upper bound and the Burstein-Moss conduction-band shift are $\approx 0.25-0.30 \mathrm{eV}$ above the Kohn-Sham conduction-band edge, there is an abrupt change in the slope of the upper bound, and the bound increasingly deviates from the Burstein-Moss conduction-band shift as the density increases further. This deviation occurs because the calculation for the Burstein-Moss conduction-band shift considers only the energy well centered at the $\Gamma$-point in the Brillouin zone and does not take into account an additional energy well centered at the Lpoint in the Brillouin zone. Consistent with both Fig. 1 and our explanation, our DFT calculations find that the L-point well lies $0.335 \mathrm{eV}$ above the $\Gamma$-point well. Once the $\Gamma$ -

point well is filled to within $\sim \mathrm{kT}=0.026 \mathrm{eV}$ of the L-point well, additional electron density begins to also fill the L-point well, causing the upper bound to deviate from the Burstein-Moss conduction band shift. In contrast, even at the largest hole densities explored in our calculations, the valence band density of states is dominated by the $\Gamma$ point, and we find good agreement between the lower bound and the Burstein-Moss valence band shift.

\subsection{As antisite in GaAs}

In this section, we consider DFT results for the technologically important As antisite $\left(\mathrm{As}_{\mathrm{Ga}}\right)$ in GaAs, and interpret them using the bounds defined in Section 3.3. From experiments, $\mathrm{As}_{\mathrm{Ga}}$ is known to have three charge states, $q=0,+1,+2$, and two levels, $0 /+1$ and $+1 /+2$, located 0.77 and $0.54 \mathrm{eV}$ above the VBE. ${ }^{42}$ DFT calculations were 
performed for these three charge states, as well as two additional ones, $q=-1$ and +3 , in order to see whether or not the bounds will identify them as band-edge-related charge states. The DFT calculations were performed in 216-, 512-, and 1000-atom supercells with $\mathrm{As}_{\mathrm{Ga}}$ in a tetrahedral structure ( $\mathrm{T}_{\mathrm{d}}$ symmetry). The defect levels were converged to within $5 \mathrm{meV}$ with respect to Brillouin-zone sampling, and test calculations were performed to verify that the levels were converged with respect to the energy cutoff (40 Ryd) for the plane wave expansion of the Kohn-Sham orbitals.

The bounds and the defect levels are plotted in Fig. 2 as a function of the inverse cube root of the supercell volume, $1 / L$. Defect levels were computed from DFT total energies using Eq. 3. Also shown are Makov-Payne fits to the $0 /+1$ and $+1 /+2$ levels. Least-squares fits of the $0 /+1$, and $+1 /+2$ levels were performed using the Makov-Payne expression ${ }^{43}$ with the $A_{1}$ term fixed at $\alpha e^{2}\left[(q-1)^{2}-q^{2}\right] / 2 \varepsilon$, where $\alpha=2.8372$ is the Madelung constant for a simple cubic lattice, $e^{2}=2$ Ryd, and $\varepsilon=12.8$ is the static dielectric constant of GaAs at room temperature. ${ }^{44}$ At infinite supercell size, the MakovPayne fits give a $0 /+1$ level at $0.35 \mathrm{eV}$ and $\mathrm{a}+1 /+2$ level at $0.11 \mathrm{eV}$ above the KohnSham VBE eigenvalue $\varepsilon_{V B E}^{B}$. Since there is no direct relationship between $\varepsilon_{V B E}^{B}$ and the experimental valence band edge, we cannot compare our calculated levels with the measured levels without applying one of the various procedures for aligning the defect levels to the experimental band edges. However, we can compare the separation between these two levels $(0.22 \mathrm{eV})$ with that of the measured levels $(0.23 \mathrm{eV}) .{ }^{42}$ The agreement is very good.

We now consider the other two levels in Fig. $2,-1 / 0$ and $+2 /+3$. To a good approximation, the $-1 / 0$ level is coincident with the upper bound, and the $+2 /+3$ level is 
coincident with the lower bound. From the discussion in Section 3.4, this indicates that the calculations for the -1 charge state are essentially giving a neutral defect with one extra electron in the conduction band of the bulk material surrounding the defect. Similarly, the calculations for the +3 charge state are giving a +2 charged defect with one electron missing from the valence band of the bulk material surrounding the defect. Thus, in agreement with experimental results, the bounds analysis of the calculations indicates that -1 and +3 are band-edge-related charge states of $\mathrm{As}_{\mathrm{Ga}}$.

\subsection{Si vacancy in silicon}

In this section, we consider new DFT results for the $0 /+1$ and $+1 /+2$ levels of the $\mathrm{Si}$ vacancy $\left(\mathrm{V}_{\mathrm{Si}}\right)$ together with previously published ${ }^{29}$ DFT results for these levels. The previously published results were obtained using nominal 216-, 512-, and 1000-atom supercells, while the new results used nominal 1728-, 2744-, and 4096-atom supercells with all other technical details of the calculations unchanged from those used in the previous study. The bounds for silicon were calculated using the same procedures noted in Section 3.3 with the technical details of the DFT calculations given in Ref. 29 .

The bounds and calculated defect levels are plotted in Fig. 3 as a function of the inverse cube root of the supercell volume, 1/L. Also shown are Makov-Payne fits of the results obtained from the 216-, 512-, and 1000-atom supercells. As reported previously, ${ }^{29}$ the fact that the $+1 /+2$ level is higher than the $0 /+1$ level means that $\mathrm{V}_{\mathrm{Si}}$ displays negative-U behavior in $p$-type material. Furthermore, the difference between the two

levels, $0.70 \mathrm{eV}$, is in good agreement with the experimental result, $0.80 \mathrm{eV} .{ }^{45}$ Also plotted in Fig. 3 are the levels obtained from the 1728-, 2744-, and 4096-atom supercells 
(which were not used in the Makov-Payne fits). These new results for the $0 /+1$ level are seen to deviate from the Makov-Payne fit and coincide with the lower bound. This indicates that for supercell sizes greater than 1000-atoms, the calculations for the +1 charge state are giving a neutral defect with one electron missing from the valence band

of the bulk material surrounding the defect. This problem with the +1 charge state calculations for supercells containing more than 1000 -atoms is also reflected in the $+1 /+2$ level, which is seen to deviate from the Makov-Payne fit at these supercell sizes. Thus, this analysis demonstrates a previously unknown aspect of DFT defect calculations, namely that using too large of a supercell in DFT defect calculations can lead to incorrect results because of the consequential supercell-size dependences of both the defect levels themselves and the bounds. In fact, in the limit of an infinite sized supercell, DFT calculations with semilocal exchange and correlation would predict that +1 and +2 are not localized defect charge states, in disagreement with experimental data.

\subsection{As interstitial in GaAs}

In this section, we consider DFT results for the As interstitial $\left(\mathrm{I}_{\mathrm{As}}\right)$. DFT calculations were performed for a tetrahedral $\left(T_{d}\right.$ symmetry) $I_{A s}$ structure surrounded by four nearestneighbor $\mathrm{Ga}$ atoms using the supercell sizes, convergence criteria, and technical parameters noted in Section 4.1. The valid charge states were found to be $q=-1,0,+1$, +2 , and +3 and thus the levels were found to be $-1 / 0,0 /+1,+1 /+2$, and $+2 /+3$. (To our knowledge, no experimental information is available for the charge states and levels of $\mathrm{I}_{\mathrm{As}}$ in GaAs.) The calculated defect levels are plotted in Fig. 4 as a function of the 
inverse cube root of the supercell volume, $1 / L$. Also plotted are Makov-Payne fits of the levels. Two features are worth noting:

First, eight out of the twelve calculated levels are above the CBE, and the highest of these is the $-1 / 0$ level in the 216-atom supercell, which is almost $0.2 \mathrm{eV}$ above the CBE. In cases such as this where the levels begin to approach the bounds, it is possible for calculations to give an intermediate situation in which an extra electron (hole) is partially localized on the defect and partially delocalized in conduction (valence) band states. Partial occupation of conduction band states corresponds to a Fermi level in the DFT calculations that is above the CBE. Likewise, partial de-occupation of the valence band states corresponds to a DFT Fermi level that is below the VBE. In such cases, the amount of occupation (de-occupation) can be estimated by first noting the Fermi level in the defect calculation, and then performing a series of calculations in a bulk supercell of the same size while varying the excess charge $-\delta(+\delta)$ until the Fermi level of the bulk calculation is equal to Fermi level of the defect calculation. We have applied this procedure to the -1 charge state in the 216-atom supercell, and we estimate the occupation of conduction-band orbitals to be 0.08 . Thus, in the transition from the 0 charge state to the -1 charge state, approximately $92 \%$ of the added charge is associated with the defect and $8 \%$ is associated with the bulk material surrounding the defect. These results demonstrate that even in the case of partial delocalization, the defect level can be well above the $\mathrm{CBE}$ without the loss of charge from the defect becoming large enough to produce major changes in defect behavior.

Secondly, we note that the energy range spanned by the extrapolated levels is 0.59 $\mathrm{eV}$, while the range spanned by the levels in the 216-atom supercell is $0.23 \mathrm{eV}$. This 
indicates that the spurious electrostatic interactions between periodically repeated 216atom defect supercells (the interactions modeled by the Makov-Payne equation ${ }^{43}$ ) can have the beneficial effect of compressing the energy range of defect levels (by $0.36 \mathrm{eV}$ in the 216-atom supercell), thereby allowing more charge states and a wider range of levels than if this effect was absent.

\section{Conclusions}

In this article, we have developed approximate bounds on the defect levels calculated using finite supercells; moreover, we have compared these proposed bounds to DFT calculations for selected defect transitions in $\mathrm{GaAs}$ and $\mathrm{Si}$. These case-by-case comparisons for defect levels located at various positions within both the bounds and the Kohn-Sham band gap show that the bounds have significant practical consequences in helping to interpret the results of such calculations. Given the ease with which these bounds can be calculated for a given material, supercell size, Brillouin zone sampling, and exchange-correlation functional, we suggest that researchers should routinely compare their calculated defect levels to the bounds.

Such comparisons provides a powerful tool to help determine if a calculated defect level involves transitions between well-localized defect charge states or instead involves carriers in band-edge-like states. When calculated defect levels remain well-separated from the bounds for a range of supercell sizes, the results of those calculations can be safely processed using the various procedures that have been developed to remove the long-ranged interactions between defects. In this case, the infinite supercell-size limit of

the defect level can be obtained and aligned with the experimental band edges to 
determine whether the level lies within the experimental band gap. Because the procedures used to remove long range interactions generally assume localized charge distributions, the results for any supercells in which the calculated defect levels are close to a bound should not be included when applying these procedures.

In some cases, comparison of defect levels to the bounds may reveal a level that follows a bound over the entire range of supercell sizes. The correct physical interpretation of such a situation might be that $(i)$ the studied defect level does not exist within the experimental band gap; (ii) the studied defect level is shallow with one of the charge states involving a carrier in a hydrogenic defect state; or (iii) the studied defect level does exist within the experimental band gap, but a more accurate exchangecorrelation functional is needed in order to obtain information about this defect level. Although the bounds alone do not allow us to determine which of these alternative scenarios is the correct physical interpretation, the bounds do provide a clear warning that further study of such cases, perhaps using a more accurate exchange-correlation functional, is needed.

\section{Acknowledgements}

This work was performed, in part, at the Center for Integrated Nanotechnologies, a U.S. Department of Energy, Office of Basic Energy Sciences user facility. Sandia National Laboratories is a multi-program laboratory managed and operated by Sandia Corporation, a wholly owned subsidiary of Lockheed Martin Corporation, for the U.S. Department of Energy's National Nuclear Security Administration under contract DEAC04-94AL85000. 
1 W. Kohn and L.J. Sham, Phys. Rev. 140, A1133 (1965).

${ }^{2}$ H.-P. Komsa and A. Pasquarello, Phys. Rev. B 84, 075207 (2011).

${ }^{3}$ A. Alkauskas, P. Broqvist, and A. Pasquarello, Phys. Stat. Sol. B 248, 775 (2011).

${ }^{4}$ H.-P. Komsa, T. T. Rantala, and A. Pasquarello, Phys. Rev. B 86, 045112 (2012).

${ }^{5}$ P. Mori-Sanchez, A.J. Cohen, and W. Yang, Phys. Rev. Lett. 100, 146401 (2008).

${ }^{6}$ C. D. Latham, M. Alatalo, R. M. Nieminen, R. Jones, S. Oberg, and P. R. Bridden, Phys. Rev. B 72, 235205 (2005).

7 Y. Bar-Yam and J.D. Joannopoulos, Phys. Rev. B 30, 1844 (1984).

${ }^{8}$ P. Carloni, P. Blochl, and M. Parinello, J. Phys. Chem 99, 1338 (1995).

${ }_{9}^{9}$ P.A. Schultz, Phys. Rev. B 60, 1551 (1999).

${ }^{10}$ P.A. Schultz, Phys. Rev. Lett. 84, 1942 (2000).

${ }^{11}$ C.A. Rozzi, D. Varsano, A. Marini, E.K.U. Gross, and A. Rubio, Phys. Rev. B 73, 205119 (2006).

${ }^{12}$ C. Freysoldt, J. Neugebauer, and C.G. Van de Walle, Phys. Status Solidi B 248, 1067 (2011).

${ }^{13}$ C. Freysoldt, B. Grabowski, T. Hickel, J. Neugebauer, G. Kresse, A. Janotti, and C. G. Van de Walle, Rev. Mod. Phys. 86, 253 (2014).

${ }^{14}$ S. B. Zhang and J. E. Northrup, Phys. Rev. Lett. 67, 2339 (1991).

${ }^{15}$ C. G. Van de Walle, D. B. Laks, G. F. Neumark, and S. T. Pantelides, Phys. Rev. B 47, 9425 (1993).

16 J. Lento, J.-L. Mozos, and R.M. Nieminen, J. Phys.: Condens. Matter 14, 2637 (2002).

${ }_{17}$ C.W.M Castleton, A. Hoglund, and S. Mirbt, Phys. Rev. B 73, 035215 (2006).

${ }^{18}$ G. Makov and M.C. Payne, Phys. Rev. B 51, 4014 (1995). 
${ }^{19}$ M. Leslie and M.J. Gillan, J. Phys. C 18, 973 (1985).

20 A.F. Wright and N.A. Modine, Phys. Rev. B 74, 235209 (2006).

${ }^{21}$ S. Lany and A. Zunger, Phys. Rev. B 78, 235104 (2008).

${ }^{22}$ L. Kleinman, Phys. Rev. B 24, 7412 (1981).

${ }^{23}$ S.B. Zhang and J.E. Northrup, Phys. Rev. Lett. 67, 2339 (1991).

${ }^{24}$ D.B. Laks, C.G. Van de Walle, G.F. Neumark, P.E. Blochl, and S.T. Pantelides, Phys. Rev. B 45, 10965 (1992).

${ }^{25}$ J.E. Northrup and S.B. Zhang, Phys. Rev. B 47, 6791 (1993).

${ }^{26}$ A. Alkauskas, P. Broqvist, and A. Pasquarello, Phys. Rev. Lett. 101, 046405 (2008).

${ }^{27}$ P.A. Schultz, Phys. Rev. Lett. 96, 246401 (2006).

${ }^{28}$ P.A. Schultz and O.A. von Lilienfeld, Modelling Simul. Mater. Sci. Eng. 17, 084007 (2009).

${ }^{29}$ A. F. Wright, Phys. Rev. B 74, 165116 (2006).

${ }^{30}$ R.R. Wixom and A.F. Wright, Phys. Rev. B 74, 205208 (2006).

${ }^{31}$ J.P. Perdew and M. Levy, Phys. Rev. Lett. 51, 1884 (1983).

${ }^{32}$ L.J. Sham and M. Schluter, Phys. Rev. Lett. 51, 1888 (1983).

${ }^{33}$ Our DFT calculations were performed using the Socorro code, which is described in detail at http://dft.sandia.gov/socorro.

${ }^{34}$ D. M. Ceperley and B. J. Alder, Phys. Rev. Lett. 45, 566 (1980); J. P. Perdew and Y. Wang, Phys. Rev. B 45, 13244 (1992).

${ }^{35}$ See http://www.fhi-berlin.mpg.de/th/fhi98PP/. Three electrons were treated as valence $\left(4 s_{2}\right.$ and $\left.4 p_{1}\right)$ in $\mathrm{Ga}$ and five electrons $\left(4 s_{2}\right.$ and $\left.4 p_{3}\right)$ in As. Cutoff radii of 1.10, 1.30, and 2.25 Bohr were used for the $s, p$, and $d$ channels of Ga and 1.00, 1.18, and 1.80 Bohr 
were used for As. Non-linear core corrections [S.G. Louie, S. Froyen, and M. L. Cohen, Phys. Rev. B 26, 1738 (1982)] were employed for both atoms with partial core charge radii of 1.20 and 1.00 Bohr for $\mathrm{Ga}$ and As, respectively. The semilocal potentials from the fhi98PP code were converted into the Kleinman-Bylander form [L. Kleinman and D. M. Bylander, Phys. Rev. Lett. 48, 1425 (1982)] by the authors with the $s$ potentials treated as the local potentials.

${ }^{36}$ A. Alkauskas and A. Pasquarello, Phys. Rev. B 84, 125206 (2011).

${ }^{37}$ H. J. Monkhorst and J. D. Pack, Phys. Rev. B 13, 5188 (1976).

${ }^{38}$ D. D. Sell, H. C. Casey and K. W. Wecht, J. Appl. Phys. 45, 2650 (1974).

${ }^{39}$ E. Burstein, Phys. Rev. 93, 632 (1954); T. S. Moss, Proc. Phys. Soc. B 67, 775 (1954).

${ }^{40}$ J. Piprek, Semiconductor Optoelectronic Devices: Introduction to Physics and

Simulation (Academic Press, San Diego, 2008), pp. 16-20; the standard valence-band effective-mass equations for GaAs were modified to account for the case of a triply degenerate valence-band maximum resulting from our spinless DFT/LDA calculations.

${ }^{41}$ S. Zh. Karazhanov and L. C. Lew Yan Voon, Semiconductors 39, 161 (2005); from Fizika i Tekhnika Poluprovodnikov 39, 177 (2005).

${ }^{42}$ J. Lagowski, D. G. Lin, T. -P. Chen, M. Skowronski, and H. C. Gatos, Appl. Phys. Lett. 47, 929 (1985).

${ }^{43}$ G. Makov and M. C. Payne, Phys. Rev. B 51, 4014 (1995). The analytic expression for $A_{1}$ was first derived by M. Leslie and M. J. Gillan and published in J. Phys. C 18, 973 (1985).

${ }_{44}$ J. S. Blakemore, J. Appl. Phys. 53, R123 (1982). 
${ }^{45}$ See G. D. Watkins, in Deep Centers in Semiconductors, edited by S. T. Pantelides

(Gordon and Breach, New York, 1986), Chapter 3 and references therein for a thorough discussion of experimental results for $\mathrm{V}_{\mathrm{Si}}$. 


\section{Figure Captions}

Figure 1: (1: filled symbols and solid lines) Bounds on defect levels in GaAs (in eV) vs. the supercell size and vs. the added or removed electron density (in $\left.\mathrm{cm}^{-3}\right)$. (2: short dashed lines) Burstein-Moss shifts of the valence- and conduction-band edges in GaAs vs. carrier density. (3: long dashed lines) Kohn-Sham VBE and CBE.

Figure 2: (1: solid lines) Spline fit to the bounds on defect levels in GaAs (in eV) vs. the inverse cube root of the supercell volume, 1/L (1/Bohr). (2: filled circles) Calculated As antisite $\left(\mathrm{As}_{\mathrm{Ga}}\right)$ levels for the $-1 / 0,0 /+1+1 /+2$, and $+2 /+3$ transitions in $216-, 512-$, and 1000-atom supercells vs. the cube root of the supercell volume. (3: dashed lines) MakovPayne fits of the calculated $0 /+1$ and $+1 /+2$ levels vs. the cube root of the supercell volume.

Figure 3: (1: solid lines) Spline fit to the bounds on defect levels in $\mathrm{Si}$ (in eV) vs. the inverse cube root of the supercell volume, 1/L (1/Bohr). (2: filled circles) Calculated $\mathrm{Si}$ vacancy $\left(\mathrm{V}_{\mathrm{Si}}\right)$ levels for the $0 /+1$ and $+1 /+2$ transitions in 216-, 512-, and 1000-atom supercells vs. the cube root of the supercell volume. (3: dashed lines) Makov-Payne fits of the levels in 216-, 512-, and 1000-atom supercells vs. the cube root of the supercell volume. (4: filled diamonds) Calculated $\mathrm{V}_{\mathrm{Si}}$ levels for the $0 /+1$ and $+1 /+2$ transitions from 1728-, 2744-, and 4096-atom supercells vs. the cube root of the supercell volume (not included in the Makov-Payne fits). 
Figure 4: (1: solid lines) Spline fit to the bounds on defect levels in GaAs (in eV) vs. the inverse cube root of the supercell volume, 1/L (1/Bohr). (2: filled circles) Calculated As interstitial $\left(\mathrm{I}_{\mathrm{As}}\right)$ levels for the $-1 / 0,0 /+1+1 /+2$, and $+2 /+3$ transitions in 216-, 512-, and 1000-atom supercells vs. the cube root of the supercell volume. (3: dashed lines) MakovPayne fits of the calculated levels vs. the cube root of the supercell volume. 
Figure 1:

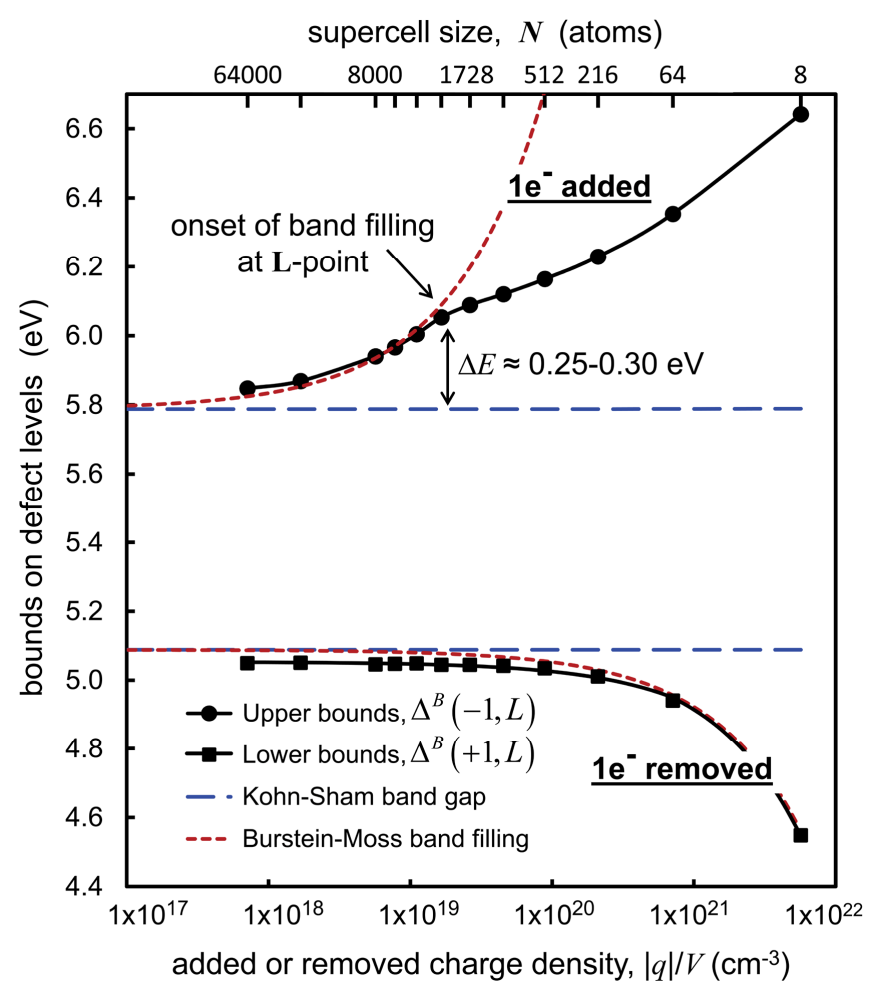


Figure 2:

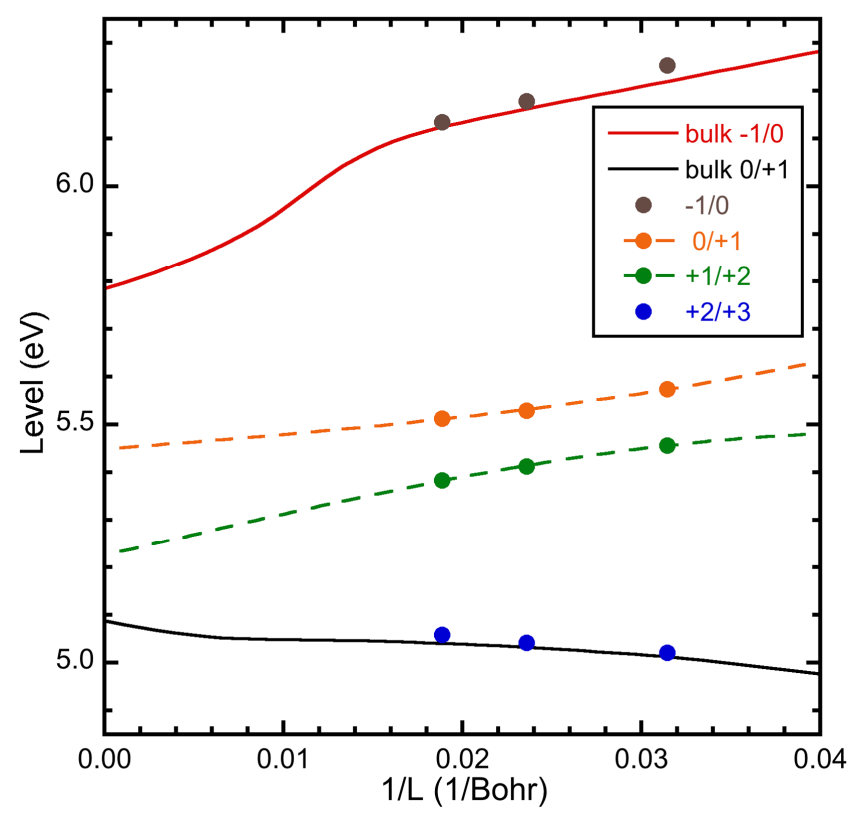


Figure 3:

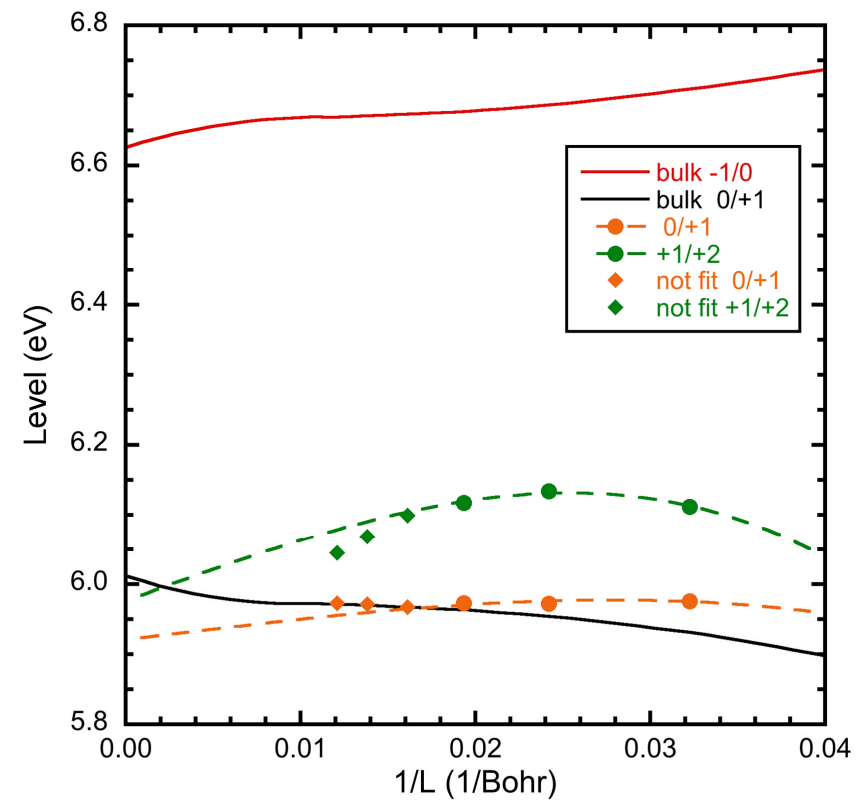


Figure 4:

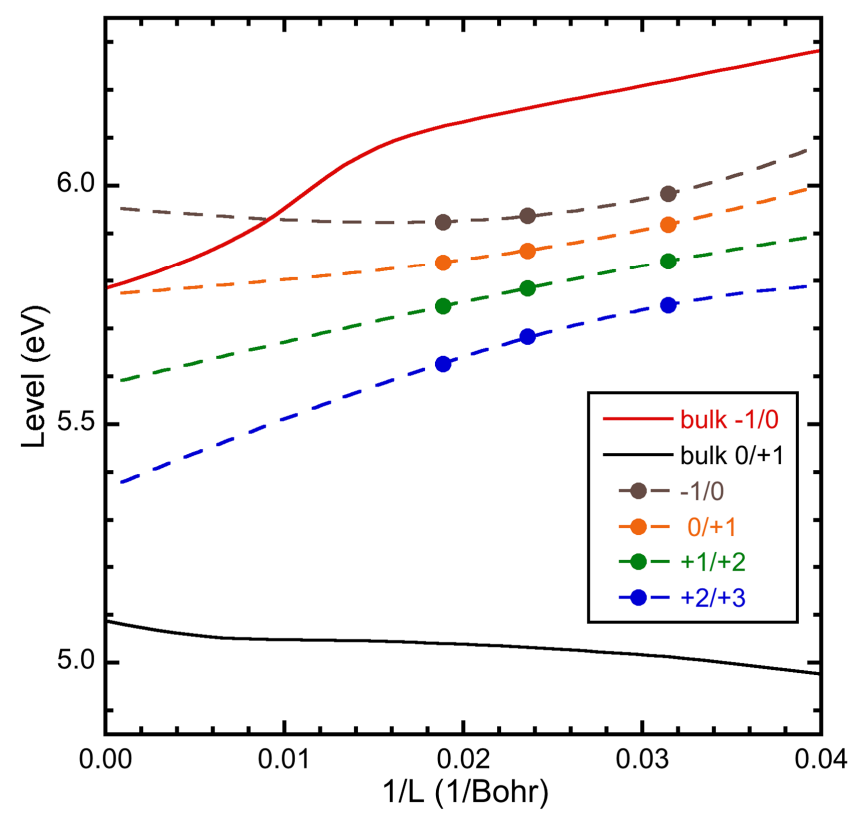




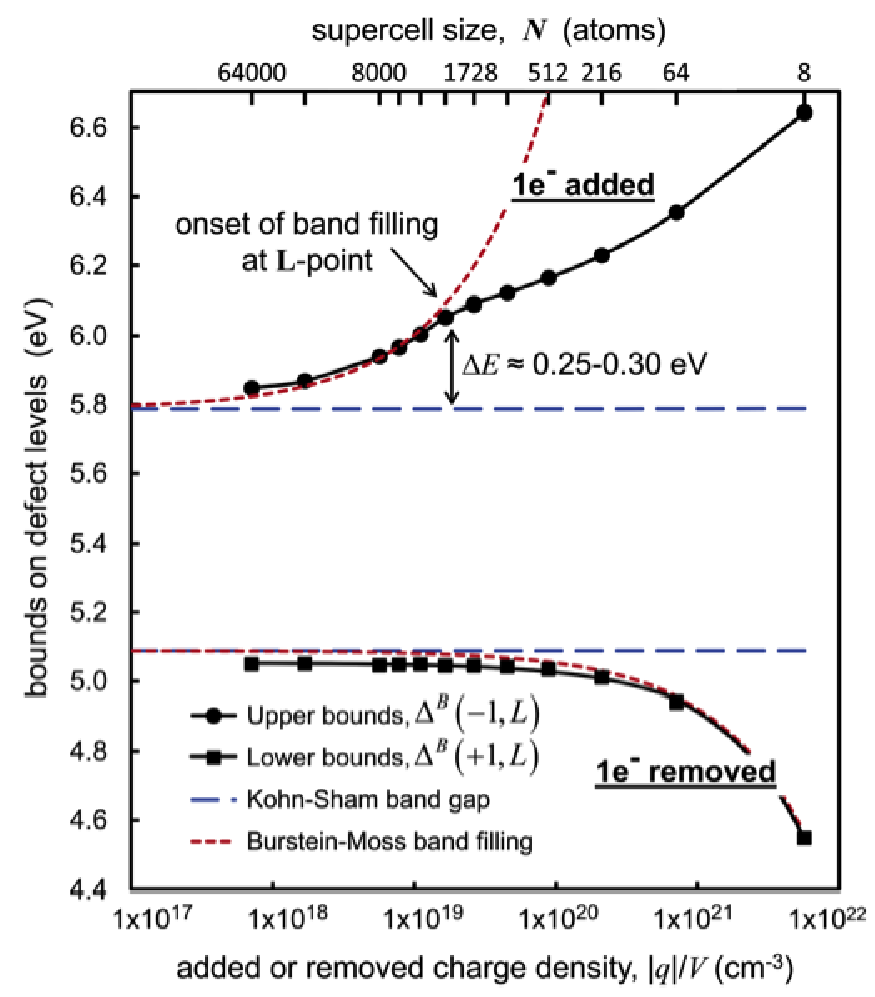

